\title{
Photoinduced renormalization and electronic screening of quasi-two-dimensional Dirac states in $\mathrm{BaNiS}_{2}$
}

\author{
N. Nilforoushan $\odot,{ }^{1}$ M. Casula, ${ }^{2}$ M. Caputo, ${ }^{1}$ E. Papalazarou, ${ }^{1}$ J. Caillaux, ${ }^{1}$ Z. Chen,,${ }^{1,3}$ L. Perfetti, ${ }^{4}$ A. Amaricci $\odot{ }^{5}$ \\ D. Santos-Cottin, ${ }^{2}$ Y. Klein ${ }^{\circ},{ }^{2}$ A. Gauzzi ${ }^{\circ},{ }^{2}$ and M. Marsi ${ }^{1}$ \\ ${ }^{1}$ Université Paris-Saclay, CNRS, Laboratoire de Physique des Solides, 91405 Orsay Cedex, France \\ ${ }^{2}$ IMPMC, Sorbonne Université, CNRS, 4 place Jussieu, 75252 Paris, France \\ ${ }^{3}$ Synchrotron SOLEIL, Saint Aubin BP 48, Gif-sur-Yvette F-91192, France \\ ${ }^{4}$ Laboratoire des Solides Irradiés, CEA/DRF/IRAMIS, Ecole Polytechnique, CNRS, \\ Institut Polytechnique de Paris, F-91128 Palaiseau, France \\ ${ }^{5}$ CNR-IOM DEMOCRITOS, Istituto Officina dei Materiali, Consiglio Nazionale delle Ricerche, Via Bonomea 265, I-34136 Trieste, Italy
}

(Received 30 December 2019; revised 30 September 2020; accepted 16 November 2020; published 21 December 2020)

\begin{abstract}
By means of time- and angle-resolved photoelectron spectroscopy, we give evidence of a remarkable reduction of the Fermi velocity of out-of-equilibrium Dirac bands in the quasi-two-dimensional semimetal $\mathrm{BaNiS}_{2}$. This effect is accompanied by a nonrigid shift of the bands at the center of the Brillouin zone. Analytical and first-principles calculations indicate that this band renormalization is ascribed to a change in nonlocal electron correlations driven by a photoinduced enhancement of screening properties. Our results are explained by a scaling relation between inverse screening length and electronic temperature that is of general relevance for the description of out-of-equilibrium dynamics and screening effects in all layered Dirac materials.
\end{abstract}

DOI: 10.1103/PhysRevResearch.2.043397

\section{INTRODUCTION}

The recent discovery of Dirac and Weyl fermions in crystalline solids [1-5] provides a favorable experimental environment for the study of these exotic electronic states and for the development of novel electronics applications. For instance, the interaction of Dirac fermions with ultrafast light pulses is attractive in view of novel concepts of devices combining unprecedented mobility and subpicosecond response or of enabling photoinduced topological phase transitions [6-8]. The electronic band structure of these materials is characterized by several linearly dispersing bands that originate from and are protected by the interplay between real- and momentum- space properties [9-12]. Therefore, the ability to manipulate these bands is a prerequisite for any functional application of relativistic Dirac fermions.

In the present paper, we demonstrate both experimentally and theoretically that Dirac bands can be renormalized using ultrafast light pulses in the quasi-two-dimensional (quasi-2D) Dirac semimetal $\mathrm{BaNiS}_{2}$. With time-resolved angle-resolved photoemission spectroscopy (trARPES), we show that photoexcitation significantly reduces the Fermi velocities of Dirac electrons. This remarkable behavior is also accompanied by nonrigid, orbital dependent band shifts at the center of the Brillouin zone [13]. Both these effects are captured by our

Published by the American Physical Society under the terms of the Creative Commons Attribution 4.0 International license. Further distribution of this work must maintain attribution to the author(s) and the published article's title, journal citation, and DOI. $a b$ initio calculations, demonstrating that the key underlying mechanism is the dynamic reduction of the electron interaction range upon photoexcitation. With the help of an analytical model we show that the screening enhancement is related to the temperature increase of excited electrons, so that our results provide a framework for a general description of the effects of screening in quasi-2D Dirac materials.

The ultrafast renormalization of the band structure has so far been investigated only in insulating phases [14-17] or in thin films where the substrate significantly affects the screening properties $[18,19]$. The present study on $\mathrm{BaNiS}_{2}$ demonstrates that ultrafast light pulses alter dramatically the fermiology and the transport properties of Dirac semimetals.

\section{ARPES EVIDENCE OF ULTRAFAST RENORMALIZATION OF DIRAC STATES}

In our experiment, $\mathrm{BaNiS}_{2}$ single crystals were cleaved in ultrahigh vacuum on the $a-b$ plane at the temperature of $130 \mathrm{~K}$. The trARPES measurements were performed by pumping with $1.55-\mathrm{eV}$ infrared pulses and by probing photoelectrons with 6.28-eV ultraviolet pulses [20] [see Fig. 1(a)]. The time resolution is $80 \mathrm{fs}$. The equilibrium Fermi surface consists of a fourfold-symmetric Dirac cone along $\Gamma-M$, and by a small electron pocket (EP) at $\Gamma$. Both structures are visible in the top panel of Fig. 1(b). The $d$-orbital nature of each band [13] is indicated in the figure legend. The photoexcited band structure is shown in the bottom panel for $\Delta t=250 \mathrm{fs}$.

In Fig. 1(c) we present the energy distribution curves (EDC's) relative to the Dirac cone: by fitting the data with a Fermi-Dirac distribution, one can extract the electronic temperature, which yields a value $T_{e} \approx 400 \mathrm{~K}$ at $\Delta t=200 \mathrm{fs}$. 

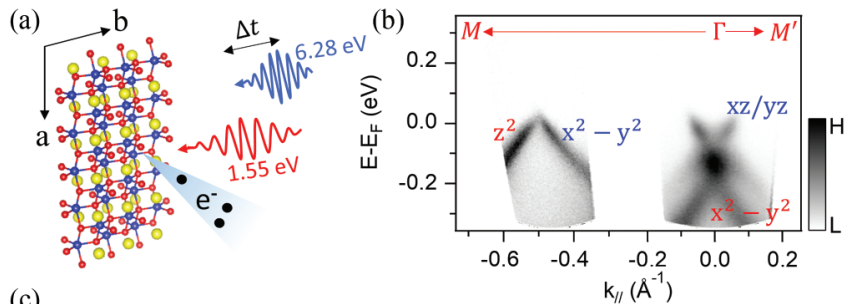

(c)
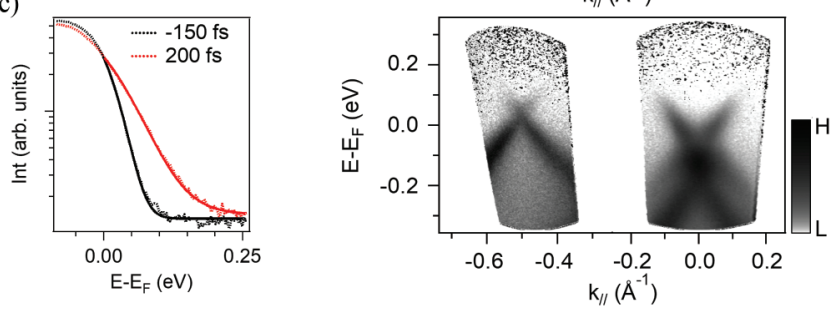

FIG. 1. (a) Experimental geometry of the trARPES setup. (b) Top: Reference spectra of the bands along $\Gamma-M$ (Dirac cone at $k_{/ /} \approx-0.5 \AA^{-1}$ and electron pocket at $\Gamma$ ). Bottom: Photoexcitation of the electronic states at a $250-\mathrm{fs}$ delay with a pump fluence of $0.2 \mathrm{~mJ} / \mathrm{cm}^{2}$. (c) Integrated EDC's around $k_{/ /}=-0.5 \AA^{-1}$ corresponding to the Dirac states: dashed lines show their photoemission spectral weight for negative and positive time delays. Solid lines are fits giving the corresponding electronic temperature $(130 \mathrm{~K}$ at negative delays; $400 \mathrm{~K}$ at $200 \mathrm{fs}$ ). Intensities are plotted in logarithmic scale.

We first focus on the ultrafast dynamics of each Dirac band by tuning the light polarization, as described elsewhere $[13,21]$. Figures 2(a) and 2(b) show the reference spectra of the $d_{z^{2}}$ and $d_{x^{2}-y^{2}}$ bands, respectively. Figures 2(c) and 2(d) show a series of difference trARPES spectra corresponding to the difference between positive and negative delay images with a pump fluence of $0.2 \mathrm{~mJ} / \mathrm{cm}^{2}$. The difference spectra show an intensity gain (red) above the Fermi level due to the photoexcitation of electrons to the unoccupied states. As illustrated in Fig. 2(g), these states reach a maximum of occupation around 250 fs due to electron scattering and impact ionization [22]. The subsequent decay dynamics occurs with a timescale of $500 \mathrm{fs}$. We now discuss the evolution of the valence bands below the Fermi level shown in Figs. 2(c) and 2(d). These states display two distinct features: (i) an expected intensity loss (shown in blue) due to a transient depletion of the electron population and (ii) a remarkable intensity gain (shown in red), a signature of an ultrafast and time-dependent renormalization, i.e., a nonrigid shift, of the Dirac states. In order to study the dynamics of the band dispersion, $E(k, t)$, for each $\Delta t$ we analyzed the momentum distribution curves (MDC's) of the ARPES yield, as reported in Appendix A. Figures 2(e) and 2(f) depict the band dispersion of the $d_{z^{2}}$ and $d_{x^{2}-y^{2}}$ band, respectively, before $(-100 \mathrm{fs})$ and after $(+250$ fs) the arrival of the pump pulse. We clearly observe an energy-dependent band shift corresponding to a reduction of Fermi velocity. This renormalization is purely photoinduced and is different from a thermal effect (see Appendix A). The renormalization is also qualitatively different from the effects of chemical substitution, even though the available data allow a comparison only with heavy Co doping [21]. In the right panel of Figs. 2(e) and 2(f), we plot the band renormalization, e.g., the change in the wave vector at the given binding energy, for different time delays. Note that the nonrigid shift of the wave vector is larger as one moves away from the Fermi level; $\Delta k$ even exceeds $0.01 \AA^{-1}$ for the $d_{x^{2}-y^{2}}$ band. In contrast, within our experimental resolution, the Fermi wave vector does not exhibit any shift and remains in its equilibrium position for all time delays. Figure 2(h) shows the dynamics of the Fermi velocity of both bands with a large reduction up to $30 \%$ for $\Delta t \sim 250$ fs. To the best of our knowledge, such

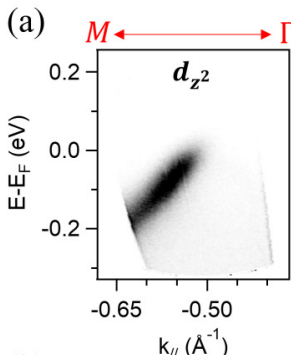

(b)

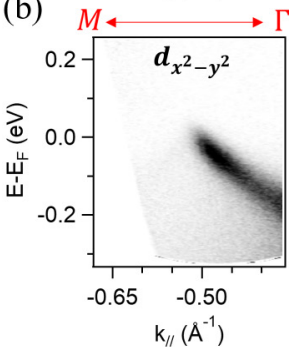

(c)

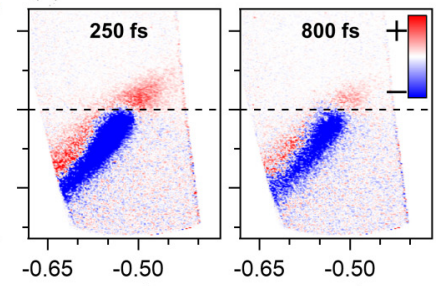

(d)

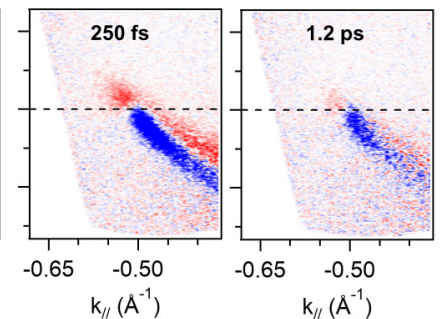

(e)

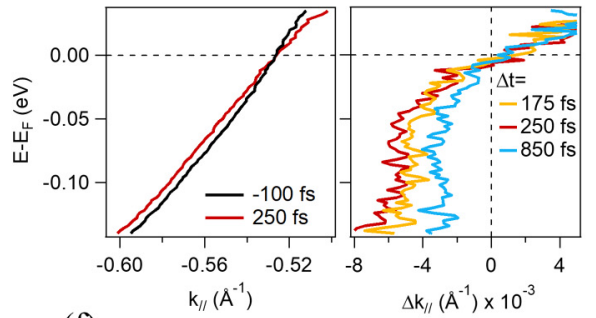

(f)

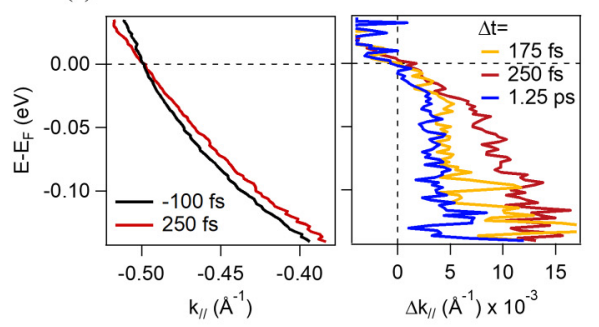

(g)

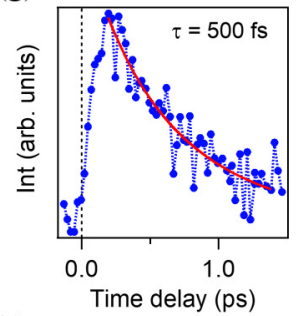

(h)

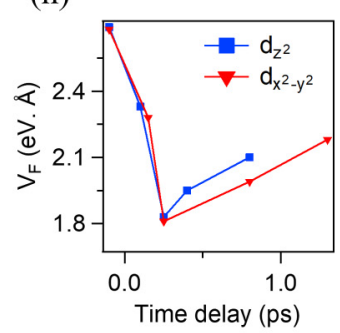

FIG. 2. ARPES reference image for the $d_{z^{2}}$ (a) and $d_{x^{2}-y^{2}}$ (b) bands. Difference trARPES images (after photoexcitation minus before photoexcitation) for each linear band are shown in (c) and (d): red and blue in the color scale indicate gain and loss of photoemission yield, respectively. Panels (e) and (f) show the band dispersion $E(k)$ of the $d_{z^{2}}$ and $d_{x^{2}-y^{2}}$ bands before and after arrival of the pump pulse, respectively. The photoinduced band renormalization for different time delays is depicted on the right: the curves are obtained by subtracting the $E(k)$ curves at the negative delay from the $E(k)$ curves at positive delays. In both cases, the photoinduced renormalization effect reaches a maximum around $250 \mathrm{fs}$. (g) Dynamics of the excited-state photoemission yield, integrated above the Fermi level. The decay takes place with a time constant of 500 fs. (h) Dynamics of the Fermi velocity for the linearly dispersing bands. 

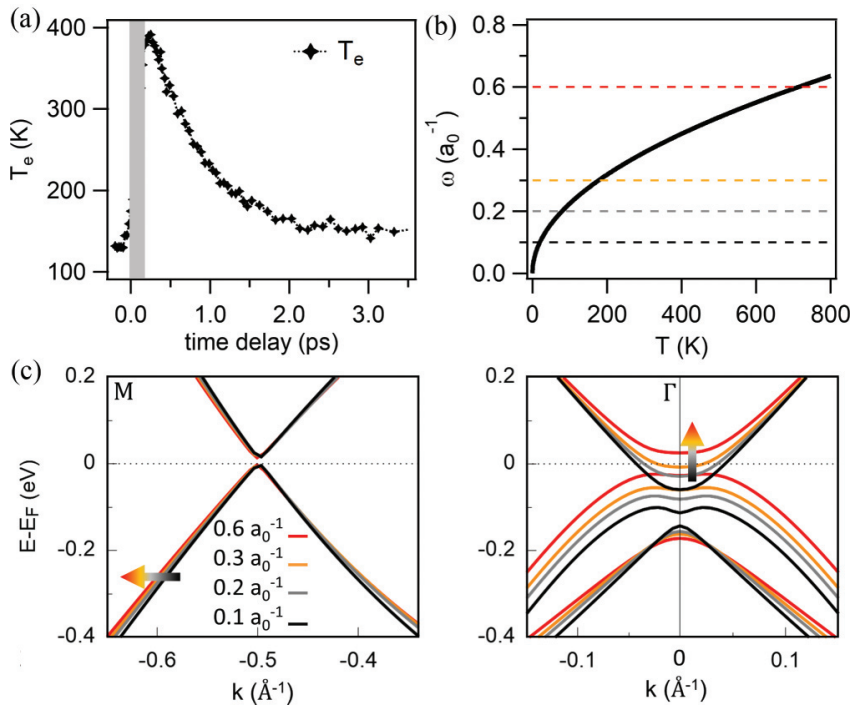

FIG. 3. (a) Temperature of hot excited electrons in the Dirac states as obtained by analyzing trARPES spectra. The gray window shows the time interval for which the temperature is ill defined, since the system cannot be described by a Fermi-Dirac distribution. (b) Inverse screening length, $\omega$, as a function of temperature. Dashed horizontal lines display the values of the inverse screening length chosen in our $a b$ initio calculations. (c) Band structure along $\Gamma-M$ computed by the hybrid HSE06 DFT functional with modified nonlocal interaction range, $\lambda=1 / \omega$. Dirac states and bands at $\Gamma$ are shown in the left and right panel, respectively. The arrows depict the effect of the increase of screening on the electronic bands. The original HSE06 value ( $\omega=0.108 a_{0}^{-1}$ ) is valid for the system at equilibrium (black lines). In the photoexcited regime with $0.2 \mathrm{~mJ} / \mathrm{cm}^{2}$ prior to relaxation, $\omega$ falls approximately in between $0.3 a_{0}^{-1}$ (orange lines) and $0.6 a_{0}^{-1}$ (red lines).

dramatic photoinduced renormalization of the band structure in a semimetal, far from any electronic or structural phase transition, has not been reported before. The temperature $T_{\mathrm{e}}$ of hot electrons, displayed in Fig. 3(a), is deduced by analyzing the evolution of the spectral weight of the Dirac states, as described in Ref. [23].

\section{THEORY OF TEMPERATURE-DEPENDENT SCREENING IN A LAYERED-DIRAC MATERIAL}

Recent out-of-equilibrium band-structure calculations [24-27], applied so far only to rather simple systems, illustrate that the electronic changes induced by a light pulse are as follows: (i) change of band occupancies, (ii) band shifts, and (iii) electronic screening variations. The latter feature varies significantly upon electron excitations. For instance, in a metal, the Debye-Hückel theory [28] predicts that, at high temperatures, the inverse screening length $\omega=1 / \lambda$ decays as $1 / \sqrt{k_{B} T_{\mathrm{e}}}$, where $k_{B}$ is the Boltzmann constant. Thus, in the conventional metallic case, the screening deteriorates as temperature increases. In contrast, the screening properties of an insulator are expected to improve when electron-hole pairs are created $[25,27]$. $\mathrm{BaNiS}_{2}$ does not fall in either case due to its marked semimetallic character given by the four Dirac cones and by its quasi-2D nature. These rather simple ingredients allow us to compute its screening properties in an analytic way by using a layered conical 2D Hamiltonian, where the nonlocal interaction between layers is retained. By deriving the dielectric function of this model in the ThomasFermi approximation (see Appendix B), we demonstrate that, in the intrinsic (i.e., undoped) regime, the inverse screening length reads

$$
\omega_{\text {Dirac }}=\sqrt{4 \log (2) \frac{e^{2} g_{S} g_{C}}{\kappa_{0} \gamma^{2} I_{d}} k_{B} T_{\mathrm{e}}}
$$

where $g_{S}$ is the spin multiplicity, $g_{C}$ the number of cones in each layer, $\kappa_{0}$ the dielectric constant of the medium, $\gamma$ the Fermi velocity, and $I_{d}$ the interlayer distance. Thus, by virtue of the reduced phase space of the Dirac band structure, $\omega_{\text {Dirac }} \propto \sqrt{k_{B} T_{\mathrm{e}}}$, which strongly differs from the conventional Debye-Hückel behavior. For $\mathrm{BaNiS}_{2}$, one obtains [29] the function plotted in Fig. 3(b). This relates $T_{\mathrm{e}}$ of the photoexcited electrons-measured as a function of the time delay [Fig. 3(a)] - to the effective $\omega_{\text {Dirac }}$ in the material. Therefore, the screening enhancement is maximum when unoccupied states close to the Fermi level are maximally populated, then it progressively decays as the system relaxes to equilibrium. This dynamics can be mapped into equilibrium $a b$ initio calculations, provided the screening parameters are modified accordingly. This approximation is justified in presence of a slow relaxation rate as indeed observed in the Dirac states [Fig. 2(g)]; the electrons are already thermalized before the band renormalization reaches its maximum. To implement these calculations, the Heyd, Scuseria, and Ernzerhof (HSE) functional [30-32] is particularly convenient, for it modifies the Perdew, Burke, and Ernzerhof (PBE) [33,34] exchange-correlation $(x c)$ functional by the addition of the screened interaction. This interaction is treated at the Fock level $\left(E_{x}^{\mathrm{HF}, \text { screened }}\right)$, such that the resulting functional reads as $E_{x c}^{\mathrm{HSE}}=E_{x c}^{\mathrm{PBE}}+\alpha\left[E_{x}^{\mathrm{HF} \text {, screened }}(\omega)-E_{x}^{\mathrm{PBE} \text {, screened }}(\omega)\right]$. In our case $\alpha=0.07$, as determined in $\mathrm{BaNiS}_{2}$ by comparison with quantum oscillation measurements [35]. The screened interaction is written as $V^{\text {screened }}(r)=\operatorname{erfc}(\omega r) / r$, where erfc is the complementary error function. For the system at equilibrium, $\omega=0.108$ in atomic units, i.e., the HSE regular value. In the case of a photoexcited system, $\omega$ increases and reaches its maximum when photoexcited electrons reach their maximum temperature as plotted in Fig. 3(b).

We performed $a b$ initio fully relativistic calculations using the modified HSE functional for $\alpha=0.07$ and $\omega=$ $\{0.108,0.2,0.3,0.6\}$ implemented in the QUANTUM ESPRESSO package [36,37]. Details of the calculations are explained in Appendix C. The calculated low-energy band structure along the $\Gamma-M$ direction is shown in Fig. 3(c). The full bandwidth is reduced by the screening enhancement, which contrasts the well-known tendency of the Coulomb exchange to widen the bandwidth [38]. According to our calculations, photoexcitation reduces the Fermi velocities by $\approx 10 \%$, in both Dirac cone branches of $d_{z^{2}}$ and $d_{x^{2}-y^{2}}$ character. Experimentally, the Fermi velocity reduction is even larger and reaches $30 \%$ [Fig. 2(h)] for both sides of the cone. This discrepancy is attributed to the dynamical nature of photoexcitation and to further correlation effects beyond the screened exchange, neglected in our scheme. 


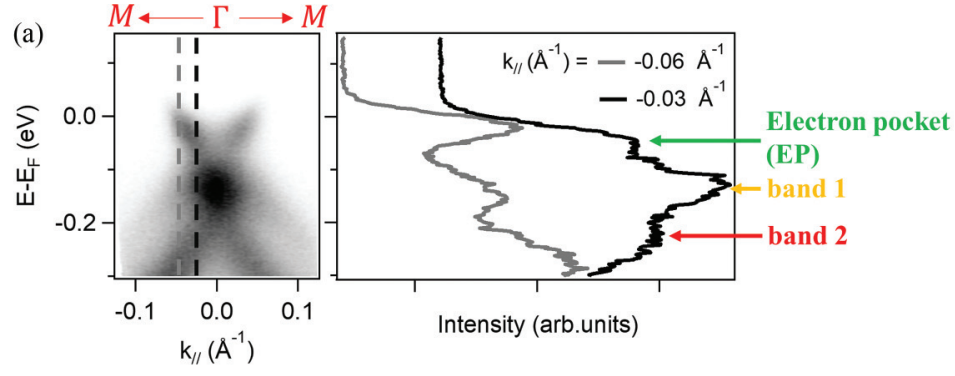

(c)

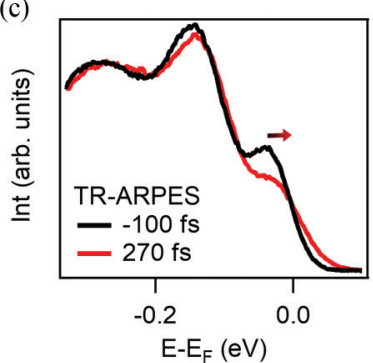

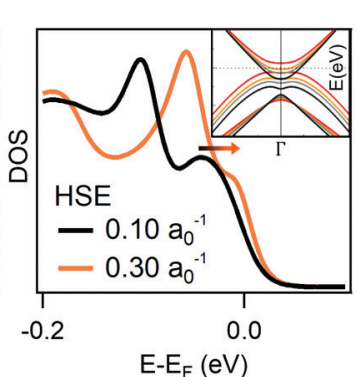

(d)

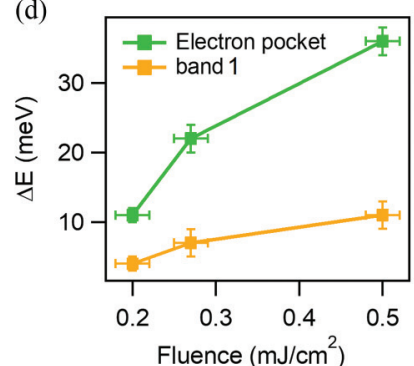

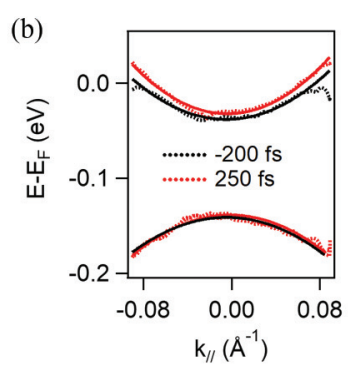

(e)

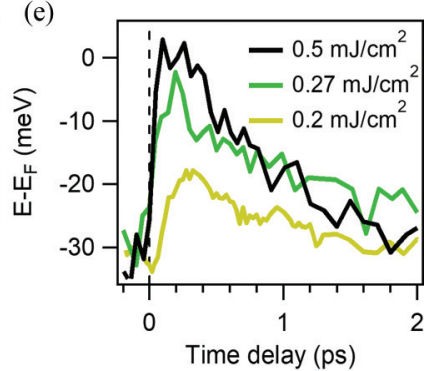

FIG. 4. (a) The ARPES spectrum before the arrival of the pump obtained with s-polarized light. The right panel shows the corresponding EDC's for several wave vectors. Arrows indicate the contribution of each of the three bands to the total intensity. We follow the binding energy of the EP and band 1 for each wave vector at different time delays. In (b) we display the band dispersion of these bands before and after photoexcitation. The solid lines are guides to the eyes. The electron pocket shows a larger shift toward lower energies with respect to band 1 . (c) Photoemission intensity integrated in a $k$ interval of $0.2 \AA^{-1}$ around $\Gamma$. The arrow shows the direction of the shift for the electron pocket that corresponds to an increase of screening effects. This is reproduced by modified HSE calculations and depicted in the right panel for two different inverse screening lengths. (d) The maximum photoinduced shift of the EP and the adjacent band (band 1) are shown as a function of the pump fluence. The error bars are estimated from the experiments. The gap separating the EP and band 1 increases with excitation density. (e) Dynamics of the center of the electron pocket for various fluences.

\section{OUT-OF-EQUILIBRIUM DYNAMICS OF THE BAND STRUCTURE}

In order to further verify the agreement between theory and experiment, we analyze the expected behavior of the band structure around $\Gamma$ upon photoexcitation [Fig. 3(c)]. In the enhanced screening regime, the electron pocket at $\Gamma$ disappears as the generating band is shifted above the Fermi level. This subtle effect depends on the combination of enhanced screening and spin-orbit coupling, which splits the set of three bands just below the $\Gamma$ point in the system at equilibrium. The bottom most band is the least affected by the screening modification and keeps almost the same position in energy.

The experimental results presented in Fig. 4 confirm that photoexcitation affects not only the velocity of the Dirac states but also the energy levels of the bands at $\Gamma$. Figure 4(a) shows the ARPES spectrum at $\Gamma$ along the $\Gamma-M$ direction taken with $s$ polarization. We trace the dispersion of the bands for different time delays by analyzing the EDC's, Fig. 4(a). Three bands contribute to the photoemission intensity. We label these bands located below the Fermi level in order of decreasing binding energy as bands 2 and 1, located below $E_{F}$, and EP, the electron pocket at $\Gamma$. In Fig. 4(b), we display the band dispersion for the electron pocket and its neighboring band 1 for the negative delay and $250 \mathrm{fs}$. The larger shift of the electron pocket towards lower binding energies points to a nonrigid shift of the band structure at the $\Gamma$ point. The pocket also shows a significant renormalization with photoexcitation, as reported in Appendix D. We now analyze in detail this nonrigid shift, by focusing on the integrated EDC's in a region of $0.2 \AA^{-1}$ around $\Gamma$, as shown in Fig. 4(c). We clearly see that the shift of the band structure at $\Gamma$ matches well our modified HSE calculations (shown in the right panel) that take into account the influence of screening. Figure 4(d) displays the maximum of the photoinduced energy shift occurring at the delay of $250 \mathrm{fs}$. Note that the maximum of $\Delta E$ for the electron pocket reaches about $11 \mathrm{meV}$ for a fluence of $0.2 \mathrm{~mJ} / \mathrm{cm}^{2}$ and $36 \mathrm{meV}$ for $0.5 \mathrm{~mJ} / \mathrm{cm}^{2}$ [Fig. 4(e)]. The dynamics of the band structure reveals that while the electron pocket is highly influenced by the ultrafast photoexcitation, band 2 hardly exhibits any appreciable shift. Clearly, our experimental data give evidence of a nonrigid and fluence-dependent energy shift of the bands at the $\Gamma$ point, further confirming the validity of our scaling relation for the inverse screening length and of our ab initio approach. It is worth emphasizing that our description of screening effects has general validity for all quasi-2D systems presenting linearly dispersing bands: in particular, while Eq. (1) is not applicable to free-standing monolayers, it is valid for all layered materials, as long as an electronic temperature can be defined. For instance, in pump-probe studies of graphite [39] and of $\mathrm{ZrSiSe}$ [40], the analysis of the band-gap renormalization dynamics indicated electron-electron interactions as the origin of the gap shrinkage. This interpretation can be easily rationalized based on our screening relation with temperature, which is thus relevant not only for trARPES experiments, but in general for all outof-equilibrium studies of materials with linearly dispersing bands. 


\section{CONCLUSION}

In conclusion, we observed that ultrafast light pulses dramatically modify the electronic band structure of the quasi-2D Dirac semimetal $\mathrm{BaNiS}_{2}$. Notable effects are a reduction of the band velocity of the Dirac states and a nonrigid shift of the bands at the center of the Brillouin zone. These effects are theoretically explained by a dynamical change of the screening length of nonlocal interactions. While particularly important for the description of ultrafast dynamics in photoexcited materials, our scaling relation with temperature presents general relevance for the interpretation of screening effects in quasi-2D Dirac systems.

\section{ACKNOWLEDGMENTS}

N.N., M.C., A.A., and M.M. acknowledge useful discussions with M. Fabrizio. M.C. acknowledges the Grand Équipement National de Calcul Intensif for providing the computational time used to carry out the calculations presented in this paper, through Project No. 0906493. This work was supported by the Region Ile-de-France (DIM OxyMORE), by the EU/FP7 under the contract Go Fast (Grant No. 280555), by "Investissement d'Avenir" Labex PALM (Grant No. ANR-10-LABX-0039-PALM), by the Equipex ATTOLAB (Grant No. ANR11-EQPX0005-ATTOLAB), and by the ANR Iridoti (Grant No. ANR-13-IS04-0001).

\section{APPENDIX A: PHOTOINDUCED VS THERMAL EFFECTS ON THE DIRAC STATES}

In this Appendix, we first show the analysis of the Dirac state renormalization based on MDC's. We then prove that this
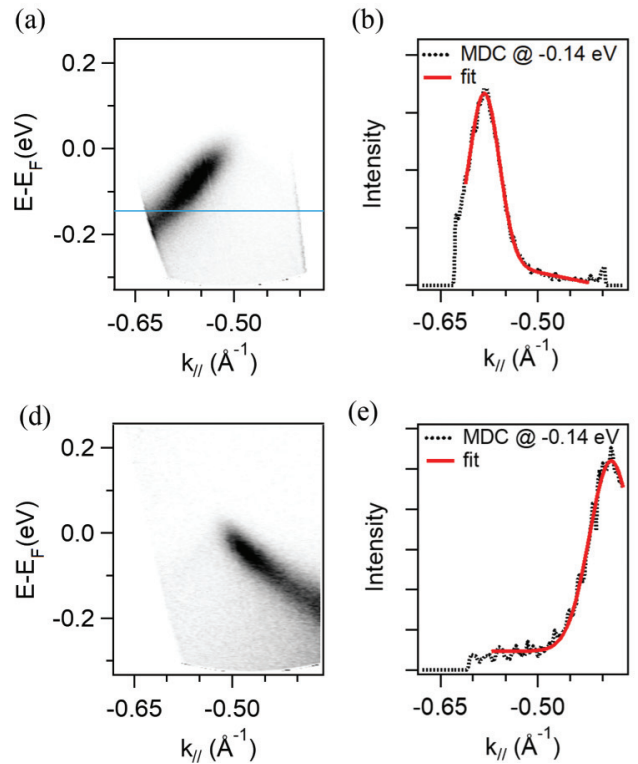

renormalization is different from a pure thermal effect and is triggered when electrons are fully decoupled from the lattice.

In order to study the chronological evolution of the band dispersion, we have sliced the ARPES yield for each time delay into MDC's, Figs. 5(b) and 5(e). The photoemission intensity peak was fitted by a Gaussian with a linear background. The center of the Gaussian shows the wave vector for each binding energy. In this way, the band dispersion can be reconstructed for each time delay $[E(k, t)]$. By stacking all of the $E(k, t)$ curves a 2D map is acquired where the intensity of each point shows the wave vector position in the $E-t$ plane, Figs. 5(c) and 5(f).

We now compare the photoinduced and thermal effects on the photoemission yield of the $d_{z^{2}}$ and $d_{x^{2}-y^{2}}$ bands, Fig. 6 . The ARPES intensities are obtained by $k$ integration around the linearly dispersing bands. The thermal induced changes are acquired by heating the sample starting from $130 \mathrm{~K}$ which is the base temperature for the time-resolved experiments. We observe that only at about $4 \mathrm{ps}$, the electronic and lattice temperatures have converged. This delay corresponds to $\Delta T \sim$ $20 \mathrm{~K}$ which corresponds to the temperature increase produced by the heat deposited by laser pulses at $0.2 \mathrm{~mJ} / \mathrm{cm}^{2}$. These data show that the renormalization of the band structure at short delays (around $200 \mathrm{fs}$ ) discussed in the paper cannot be explained by a thermal effect and is purely photoinduced.

\section{APPENDIX B: RELATION BETWEEN ELECTRONIC TEMPERATURE AND SCREENING LENGTH IN LAYERED DIRAC MATERIALS}

For an electronically excited regime, we would like to infer its impact on the screening length of the effective Coulomb interaction in $\mathrm{BaNiS}_{2}$. For simplicity, we have assumed that the excited electrons are thermalized, i.e., we can assign an
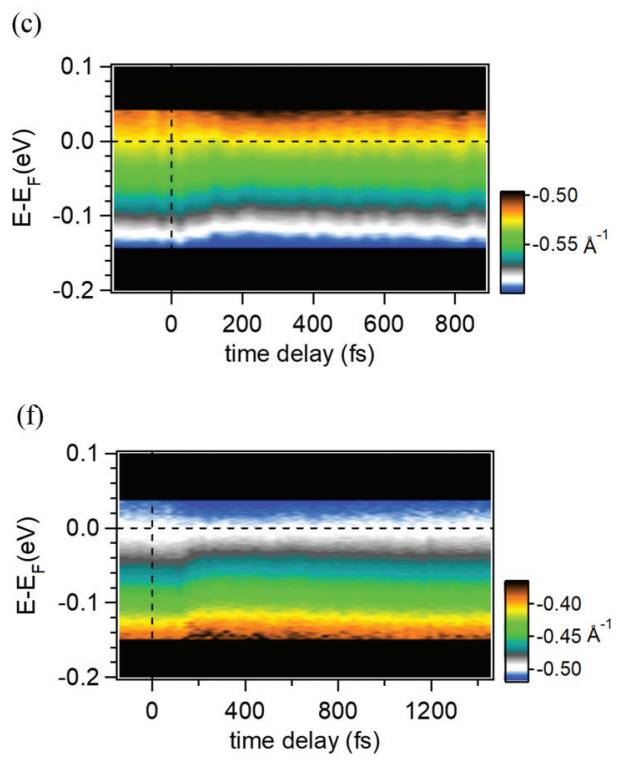

FIG. 5. Analysis of the Dirac state renormalization: (a) ARPES reference image of the $d_{z^{2}}$ band taken with the $p$ polarization of light. (b) The momentum distribution curve (MDC) at $E-E_{F}=-0.14 \mathrm{eV}$ that is shown by a blue line in the ARPES image. The red curve shows the fit with a Gaussian function and a linear base line. (c) Stacking of the reconstructed band dispersion for all delays. The intensity at each point shows the position of the wave vector in the $E-t$ plane. The bottom panel shows the same procedure for the $d_{x^{2}-y^{2}}$ band. The ARPES image is taken with the $s$ polarization. 

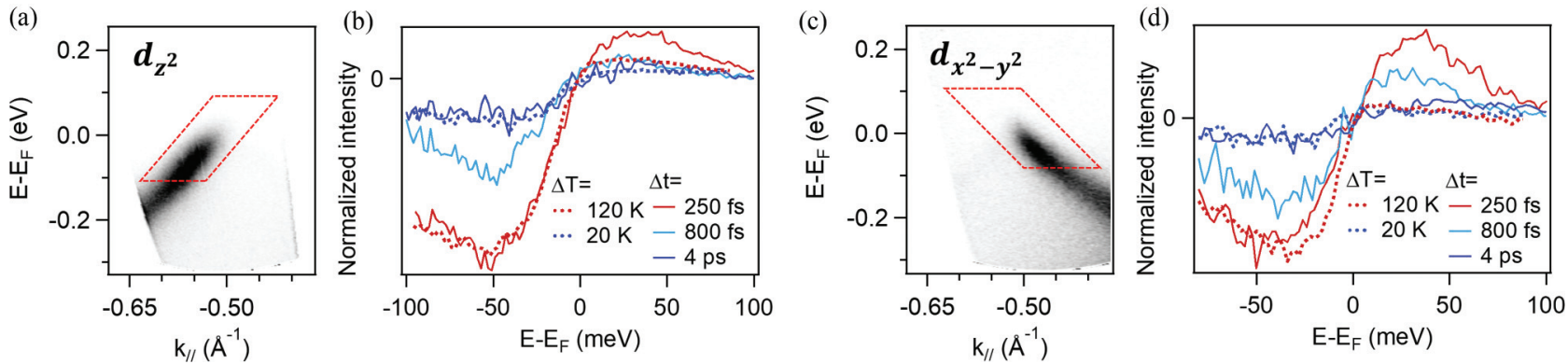

FIG. 6. (a) The dashed red contour shows the area of interest for $k$ integration around the $d_{z^{2}}$ band. (b) The spectra show the change in the photoemission yield. Solid lines show the photoinduced changes in the intensity with the pump fluence of $0.2 \mathrm{~mJ} / \mathrm{cm}^{2}(\mathrm{solid}$ lines). Dashed lines are the thermal-induced changes of the intensity while heating the sample starting from $130 \mathrm{~K}$. The electronic and the lattice temperature converge around $\approx 4$ ps corresponding to $\Delta T=20 \mathrm{~K}$. The same set of data is shown for the $d_{x^{2}-y^{2}}$ band in panels (c) and (d).

effective temperature to them at each time delay. This assumption is supported by our trARPES experiments. According to the experiment, when the magnitude of the renormalization effects is largest (at a $\approx 250$-fs delay), the electrons have already been fully thermalized. We thus derive the fundamental relation between electronic temperature and effective Coulomb interaction in a layered Dirac material, such as $\mathrm{BaNiS}_{2}$. Its semimetallic character is given by the linearly dispersing bands at the four Dirac points (at fixed $k_{z}$ ), which dominate the low-energy physics. Another relevant feature of $\mathrm{BaNiS}_{2}$ is its layered structure, with weak band dispersion along the $k_{z}$ direction. Thus, the Dirac points become Dirac lines if one takes into account the $k_{z}$ extension of the Brillouin zone in the limit of null $k_{z}$ dispersion.

With the aim of incorporating these main ingredients, we start from a conical model in two dimensions, with the Dirac Hamiltonian written as

$$
H=\gamma\left(\sigma_{x} k_{x}+\sigma_{y} k_{y}\right)-\mu,
$$

where $\mathbf{k}=\left(k_{x}, k_{y}\right)$ is the $2 \mathrm{D}$ momentum, $\sigma_{i}$ are the Pauli matrices, $\gamma$ is the Fermi velocity, and $\mu$ is the chemical potential. For $\mu=0$, the density of states of this model reads

$$
D(\epsilon)=g_{S} g_{C} \frac{|\epsilon|}{2 \pi \gamma^{2}},
$$

with $g_{S}$ the spin degeneracy, and $g_{C}$ the number of Dirac cones. This model has largely been studied in literature, mainly in the context of graphene. Here, we address a very specific question, namely, the modification of the screened Coulomb interaction driven by electronic excitations in a system made of layers, separated by a distance $I_{d}$, where the noninteracting part of every layer is described by the 2D Hamiltonian in Eq. (B1), and the hopping between layers is neglected. We assume the system to be in the so-called intrinsic (i.e., undoped, $\mu=0$ ) regime, which is appropriate for $\mathrm{BaNiS}_{2}$. The dielectric function of such a model is

$$
\epsilon\left(\mathbf{q}, k_{z}\right)=1+v^{2 D}(q) S\left(q, k_{z}\right) \Pi(q, \beta),
$$

where $\left(\mathbf{q}, k_{z}\right)$ is the three-dimensional (3D) momentum, $v^{2 \mathrm{D}}(q)=2 \pi e^{2} /\left(\kappa_{0} q\right)$ is the 2D Fourier transform of the Coulomb potential with $\kappa_{0}$ the dielectric constant of the medium, $\Pi(q, \beta)$ is the polarization function of the conical model Eq. (B1), $\beta=1 /\left(k_{B} T\right)$ is the inverse temperature $T$ with $k_{B}$ the Boltzmann constant, and $S$ takes into account the effect of interlayer interactions:

$$
S\left(q, k_{z}\right)=\frac{\sinh \left(q I_{d}\right)}{\cosh \left(q I_{d}\right)-\cos \left(k_{z} I_{d}\right)} .
$$

The latter equation has been derived by linear response theory in Ref. [41]. In Eq. (B3), we take the static limit of the dielectric function, while keeping the full temperature dependence $\beta$. This is at variance with previous studies which focused on its zero-temperature limit. We are most interested here in the impact of electronic excitations to the static screening of the quasi-2D Dirac systems.

It is well known $[42,43]$ that, in the intrinsic regime, the 2D polarization function of the conical model takes the form

$$
\Pi(q, \beta \rightarrow \infty)=\frac{g_{S} g_{C}}{16 \gamma} q .
$$

However, the zero-momentum limit is profoundly modified by thermal excitations, which give a finite value of $\Pi(q \rightarrow$ $0, \beta)$. We show in the following that this holds at any finite temperature. This result can be obtained by taking the $q \rightarrow 0$ limit of the full $\Pi(q, \beta)$ function derived in Ref. [44] through quantum field theory techniques:

$$
\Pi(q, \beta)=\frac{g_{S} g_{C}}{\pi \gamma^{2}} \frac{1}{\beta} \int_{0}^{1} d x \log \{2 \cosh [q \gamma \beta \sqrt{x(1-x)}]\} .
$$

The $q=0$ finite-temperature expression can also be derived by exploiting the compressibility sum rule:

$$
\Pi(0, \beta)=\int_{-\infty}^{\infty} d \epsilon\left(-\frac{\partial f(\epsilon)}{\partial \epsilon}\right) D(\epsilon) .
$$

From both Eqs. (B6) and (B7), one easily obtains that

$$
\Pi(0, \beta)=\frac{g_{S} g_{C}}{2 \pi \gamma^{2}} 2 \log (2) \frac{1}{\beta} .
$$

The $q \rightarrow 0$ limit of Eq. (B6) implies that Eq. (B8) holds at any order in $\beta$. Thus, it is not just a large temperature expansion. Moreover, it is also valid at finite $q$, as long as $q \lesssim \frac{16 \log (2)}{\pi} \frac{1}{\beta}$. The larger the temperature, the wider the $q$ region where Eq. (B8) is appropriate. Therefore, the screening properties and the related asymptotic behavior of the screened interaction are significantly influenced by temperature/excitation effects, as we will illustrate below. 
TABLE I. Values used to evaluate the inverse screening length $\omega_{\mathrm{TF}}$ in Eq. (B11) as a function of temperature for $\mathrm{BaNiS}_{2}$. An educated guess for the dielectric constant of the medium, $\kappa_{0}$, is taken from the value measured for sulfur [41], as the in-layer $\mathrm{Ni}$ states which make the Dirac cones in $\mathrm{BaNiS}_{2}$ are surrounded by sulfur pyramids and hybridized with sulfur $p$ states. The function $\omega_{\mathrm{TF}}=\omega_{\mathrm{TF}}(T)$ is plotted in Fig. 3(b).

\begin{tabular}{ccccc}
\hline \hline$g_{S}$ & $g_{C}$ & $\kappa_{0}$ & $\gamma(\AA \mathrm{eV})$ & $I_{d}(\AA)$ \\
\hline 2 & 4 & 3 & 2.7 & 8.908 \\
\hline \hline
\end{tabular}

To compute the dielectric function of the 3D layered system, one can plug the temperature-dependent expression for the 2D polarization function of Eq. (B8) into Eq. (B3). By using the Thomas-Fermi (TF) approach, the resulting 3D screened interaction is

$$
V_{\text {screened }}^{3 \mathrm{D}}(r)=\frac{2 e^{2}}{\pi \kappa_{0}} \int_{-\pi / I_{d}}^{\pi / I_{d}} d x K_{0}\left(r_{2 \mathrm{D}} \sqrt{x^{2}+\omega_{\mathrm{TF}}^{2}}\right) \exp \left(i l I_{d} x\right)
$$

where the 3D distance is given by $r=\sqrt{r_{2 \mathrm{D}}^{2}+\left(l I_{d}\right)^{2}}$, with $r_{2 \mathrm{D}}$ the distance on the layer, and $l I_{d}$ the out-of-plane distance, with $l$ an integer. $K_{0}$ is the modified Bessel function of the second kind. The large- $r$ asymptotic behavior of Eq. (B9) is given by

$$
V_{\text {screened }}^{3 \mathrm{D}}(r) \sim \frac{e^{2}}{\kappa_{0} r} e^{-r \omega_{\mathrm{TF}}},
$$

which has the usual Yukawa form, but this time with a TF screening parameter that reads

$$
\omega_{\mathrm{TF}}=\sqrt{4 \log (2) \frac{e^{2} g_{S} g_{C}}{\kappa_{0} \gamma^{2} I_{d}} \frac{1}{\beta}} .
$$

Therefore, the stronger the excitations, the shorter the screening length. Indeed, the inverse screening length has a $\sqrt{T}$ temperature dependence. This is very different from conventional metals, where the inverse screening length goes like $1 / \sqrt{T}$, according to the Debye-Hückel behavior in the hightemperature limit. An expression similar to Eq. (B11) has been found for nodal-line semimetals in an extrinsic hightemperature regime (i.e., $0<\mu \ll T$ ) [45]. The parameters of Eq. (B11) which are appropriate for $\mathrm{BaNiS}_{2}$ are reported in Table I.

The remarkable agreement between the band-structure modification computed from first principles and the one measured in pump-probe ARPES comes directly from the phase-space restrictions of the Dirac dispersion, which implies that in a regime of excited Dirac states the screening length is shorter than in the relaxed regime.

\section{APPENDIX C: AB INITIO CALCULATIONS}

The $\mathrm{BaNiS}_{2}$ geometry is taken from the experiment, as described by Grey and Steinfink [46]. The ab initio density functional theory calculations are done with the modified Heyd, Scuseria, and Ernzerhof (HSE06) functional [30-32], as explained in the paper, by using the QUANTUM ESPRESSO package $[36,37]$. The spin-orbit coupling is included in the $\mathrm{Ni}$
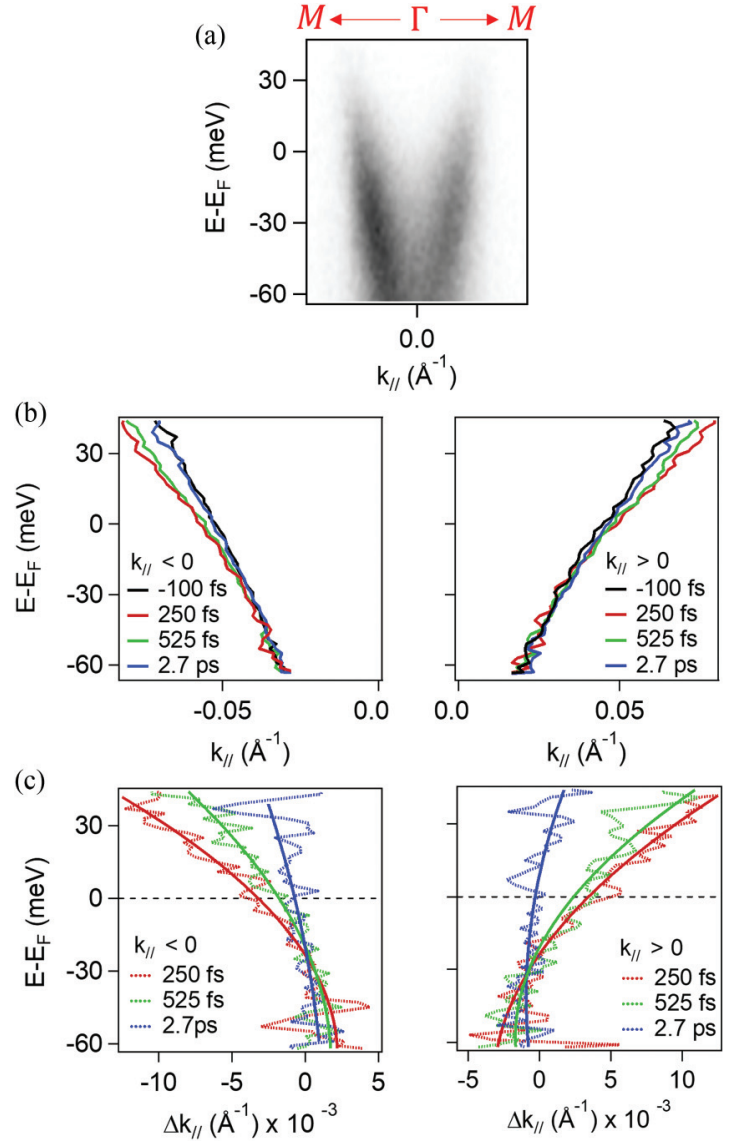

FIG. 7. (a) An enlarged spectrum of the electron pocket at equilibrium. For the sake of clarity, we show the dynamics of the bands at $k_{\|}<0$ and $k_{\|}>0$ sides separately. (b) The band dispersions obtained via MDC's are shown for before and after the arrival of the pump pulse. (c) The change in the wave vector with respect to the equilibrium state is shown for different time delays. The solid lines are guides to the eyes to highlight the general trend of the band renormalization with time. Note that $\Delta k$ is larger for lower binding energies and the Fermi velocity is reduced with photoexcitation.

norm-conserving pseudopotential in a $4 s^{2} 3 d^{8}$ atomic configuration. Norm-conserving pseudopotentials are used also to replace $\mathrm{Ba}$ and $\mathrm{S}$ atomic cores. The Ba pseudopotential includes the semicore states in valence, while $\mathrm{S}$ is in the $3 s^{2} 3 p^{4}$ configuration. The total energies are converged with a $8 \times 8 \times 8$ electron-momentum grid, a Methfessel-Paxton smearing of $0.01 \mathrm{Ry}$ for the $k$-point integration, and a plane-wave cutoff of $60 \mathrm{Ry}$ for the wave-function expansion. To compute the nonlocal Fock operator, we employed a downsampled $8 \times 8 \times 2$ $q$-point grid, with a half-a-grid shift in the $z$ direction to minimize the number of nonequivalent momenta in the $k+q$ mesh.

\section{APPENDIX D: ULTRAFAST $\boldsymbol{k}$ RENORMALIZATION at $\boldsymbol{\Gamma}$}

Here, we show that the electron pocket undergoes an ultrafast renormalization with photoexcitation. By analyzing the MDC's, we plot the band dispersion as well as the wave-vector renormalization $(\Delta k)$ for each time delay. The results are shown in Figs. 7(b) and 7(c), respectively. 
[1] N. P. Armitage, E. J. Mele, and A. Vishwanath, Weyl and Dirac semimetals in three-dimensional solids, Rev. Mod. Phys. 90, 015001 (2018).

[2] Z. K. Liu, B. Zhou, Y. Zhang, Z. J. Wang, H. M. Weng, D. Prabhakaran, S.-K. Mo, Z. X. Shen, Z. Fang, X. Dai, Z. Hussain, and Y. L. Chen, Discovery of a three-dimensional topological dirac semimetal, $\mathrm{Na}_{3} \mathrm{Bi}$, Science 343, 864 (2014).

[3] S.-Y. Xu, I. Belopolski, N. Alidoust, M. Neupane, G. Bian, C. Zhang, R. Sankar, G. Chang, Z. Yuan, C.-C. Lee, S.-M. Huang, H. Zheng, D. S. Sanchez, B. Wang, A. Bansil, F. Chou, P. P. Shibayev, H. Lin, S. Jia, and M. Z. Hasan, Discovery of a Weyl fermion semimetal and topological Fermi arcs, Science 349, 613 (2015).

[4] Z. Wang, Y. Sun, X. Q. Chen, C. Franchini, G. Xu, H. Weng, $\mathrm{X}$. Dai, and Z. Fang, Dirac semimetal and topological phase transitions in $\mathrm{A}_{3} \mathrm{Bi}(\mathrm{A}=\mathrm{Na}, \mathrm{K}, \mathrm{Rb})$, Phys. Rev. B 85, 195320 (2012).

[5] S. Borisenko, Q. Gibson, D. Evtushinsky, V. Zabolotnyy, B. Büchner, and R. J. Cava, Experimental Realization of a ThreeDimensional Dirac Semimetal, Phys. Rev. Lett. 113, 027603 (2014).

[6] Y. H. Wang, H. Steinberg, P. Jarillo-Herrero, and N. Gedik, Observation of floquet-bloch states on the surface of a topological insulator, Science 342, 453 (2013).

[7] C. P. Weber, B. S. Berggren, M. G. Masten, T. C. Ogloza, S. Deckoff-Jones, J. Madéo, M. K. L. Man, K. M. Dani, L. Zhao, G. Chen, J. Liu, Z. Mao, L. M. Schoop, B. V. Lotsch, S. S. P. Parkin, and A. Mazhar, Similar ultrafast dynamics of several dissimilar Dirac and Weyl semimetals, J. Appl. Phys. 122, 223102 (2017).

[8] E. J. Sie, C. M. Nyby, C. D. Pemmaraju, S. J. Park, X. Shen, J. Yang, M. C. Hoffmann, B. K. Ofori-Okai, R. Li, A. H. Reid, S. Weathersby, E. Mannebach, N. Finney, D. Rhodes, D. Chenet, A. Antony, L. Balicas, J. Hone, T. P. Devereaux, T. F. Heinz, X. Wang, and A. M. Lindenberg, An ultrafast symmetry switch in a Weyl semimetal, Nature (London) 565, 61 (2019).

[9] S. M. Young, S. Zaheer, J. C. Y. Teo, C. L. Kane, E. J. Mele, and A. M. Rappe, Dirac Semimetal in Three Dimensions, Phys. Rev. Lett. 108, 140405 (2012).

[10] S. M. Young and C. L. Kane, Dirac Semimetals in Two Dimensions, Phys. Rev. Lett. 115, 126803 (2015).

[11] L. Tarruell, D. Greif, T. Uehlinger, G. Jotzu, and T. Esslinger, Creating, moving and merging Dirac points with a Fermi gas in a tunable honeycomb lattice, Nature (London) 483, 302 (2012).

[12] M. S. Bahramy, O. J. Clark, B. J. Yang, J. Feng, L. Bawden, J. M. Riley, I. Markovic, F. Mazzola, V. Sunko, D. Biswas, S. P. Cooil, M. Jorge, J. W. Wells, M. Leandersson, T. Balasubramanian, J. Fujii, I. Vobornik, J. E. Rault, T. K. Kim, M. Hoesch, K. Okawa, M. Asakawa, T. Sasagawa, T. Eknapakul, W. Meevasana, and P. D. King, Ubiquitous formation of bulk Dirac cones and topological surface states from a single orbital manifold in transition-metal dichalcogenides, Nat. Mater. 17, 21 (2017).

[13] D. Santos-Cottin, M. Casula, G. Lantz, Y. Klein, L. Petaccia, P. Le Fèvre, F. Bertran, E. Papalazarou, M. Marsi, and A. Gauzzi, Rashba coupling amplification by a staggered crystal field, Nat. Commun. 7, 11258 (2016).

[14] V. Brouet, J. Mauchain, E. Papalazarou, J. Faure, M. Marsi, P. H. Lin, A. Taleb-Ibrahimi, P. Le Fèvre, F. Bertran, L. Cario, E. Janod, B. Corraze, V. T. Phuoc, and L. Perfetti, Ultrafast filling of an electronic pseudogap in an incommensurate crystal, Phys. Rev. B 87, 041106(R) (2013).

[15] J. D. Rameau, S. Freutel, L. Rettig, I. Avigo, M. Ligges, Y. Yoshida, H. Eisaki, J. Schneeloch, R. D. Zhong, Z. J. Xu, G. D. Gu, P. D. Johnson, and U. Bovensiepen, Photoinduced changes in the cuprate electronic structure revealed by femtosecond time- and angle-resolved photoemission, Phys. Rev. B 89, 115115 (2014).

[16] S. Mor, M. Herzog, D. Golež, P. Werner, M. Eckstein, N. Katayama, M. Nohara, H. Takagi, T. Mizokawa, C. Monney, and J. Stähler, Ultrafast Electronic Band Gap Control in an Excitonic Insulator, Phys. Rev. Lett. 119, 086401 (2017).

[17] S. Roth, A. Crepaldi, M. Puppin, G. Gatti, D. Bugini, I. Grimaldi, T. R. Barrilot, C. A. Arrell, F. Frassetto, L. Poletto, M. Chergui, A. Marini, and M. Grioni, Photocarrier-induced bandgap renormalization and ultrafast charge dynamics in black phosphorus, 2D Mater. 6, 031001 (2019).

[18] S. Ulstrup, A. G. Cabo, J. A. Miwa, J. M. Riley, S. S. Grønborg, J. C. Johannsen, C. Cacho, O. Alexander, R. T. Chapman, E. Springate, M. Bianchi, M. Dendzik, J. V. Lauritsen, P. D. C. King, and P. Hofmann, Ultrafast band structure control of a twodimensional heterostructure, ACS Nano 10, 6315 (2016).

[19] C. W. Nicholson, W. G. Schmidt, M. Puppin, L. Rettig, R. Ernstorfer, and M. Wolf, Beyond the molecular movie: Dynamics of bands and bonds during a photoinduced phase transition, Science 362, 821 (2018).

[20] J. Faure, J. Mauchain, E. Papalazarou, W. Yan, J. Pinon, M. Marsi, and L. Perfetti, Full characterization and optimization of a femtosecond ultraviolet laser source for time and angleresolved photoemission on solid surfaces, Rev. Sci. Instrum. 83, 043109 (2012).

[21] N. Nilforoushan, M. Casula, A. Amaricci, M. Caputo, J. Caillaux, L. Khalil, E. Papalazarou, P. Simon, L. Perfetti, I. Vobornik, P. K. Das, J. Fujii, D. Santos-Cottin, Y. Klein, M. Fabrizio, A. Gauzzi, and M. Marsi, Tuning Dirac nodes with correlated d-electrons in $\mathrm{BaNiS}_{2}$, arXiv:1905.12210.

[22] M. Caputo, L. Khalil, E. Papalazarou, N. Nilforoushan, L. Perfetti, A. Taleb-Ibrahimi, Q. D. Gibson, R. J. Cava, and M. Marsi, Dynamics of out-of-equilibrium electron and hole pockets in the type-II Weyl semimetal candidate $\mathrm{WTe}_{2}$, Phys. Rev. B 97, 115115 (2018).

[23] M. Hajlaoui, E. Papalazarou, J. Mauchain, L. Perfetti, A. TalebIbrahimi, F. Navarin, M. Monteverde, P. Auban-Senzier, C. R. Pasquier, N. Moisan, D. Boschetto, M. Neupane, M. Z. Hasan, T. Durakiewicz, Z. Jiang, Y. Xu, I. Miotkowski, Y. P. Chen, S. Jia, H. W. Ji, R. J. Cava, and M. Marsi, Tuning a Schottky barrier in a photoexcited topological insulator with transient Dirac cone electron-hole asymmetry, Nat. Commun. 5, 3003 (2014).

[24] M. Volkov, S. A. Sato, F. Schlaepfer, L. Kasmi, N. Hartmann, M. Lucchini, L. Gallmann, A. Rubio, and U. Keller, Attosecond screening dynamics mediated by electron localization in transition metals, Nat. Phys. 15, 1145 (2019).

[25] N. Tancogne-Dejean, M. A. Sentef, and A. Rubio, Ultrafast Modification of Hubbard U in a Strongly Correlated Material: $A b$ initio High-Harmonic Generation in NiO, Phys. Rev. Lett. 121, 097402 (2018).

[26] D. Golež, L. Boehnke, M. Eckstein, and P. Werner, Dynamics of photodoped charge transfer insulators, Phys. Rev. B 100 , 041111(R) (2019). 
[27] D. Golež, M. Eckstein, and P. Werner, Multiband nonequilibrium $G W+$ EDMFT formalism for correlated insulators, Phys. Rev. B 100, 235117 (2019).

[28] J.-P. Hansen and I. R. McDonald, Theory of Simple Liquids (Elsevier, Amsterdam, 1990).

[29] The full set of parameters valid for $\mathrm{BaNiS}_{2}$ is reported in Table I of Appendix B.

[30] J. Heyd, G. E. Scuseria, and M. Ernzerhof, Hybrid functionals based on a screened Coulomb potential, J. Chem. Phys. 118, 8207 (2003).

[31] J. Heyd, G. E. Scuseria, and M. Ernzerhof, Erratum: Hybrid functionals based on a screened Coulomb potential, J. Chem. Phys. 124, 219906(E) (2006).

[32] A. V. Krukau, O. A. Vydrov, A. F. Izmaylov, and G. E. Scuseria, Influence of the exchange screening parameter on the performance of screened hybrid functionals, J. Chem. Phys. 125, 224106 (2006).

[33] J. P. Perdew, K. Burke, and M. Ernzerhof, Generalized Gradient Approximation Made Simple, Phys. Rev. Lett. 77, 3865 (1996).

[34] J. P. Perdew, K. Burke, and M. Ernzerhof, Erratum: Generalized Gradient Approximation Made Simple, Phys. Rev. Lett. 78, 1396(E) (1997).

[35] Y. Klein, M. Casula, D. Santos-Cottin, A. Audouard, D. Vignolles, G. Fève, V. Freulon, B. Plaçais, M. Verseils, H. Yang, L. Paulatto, and A. Gauzzi, Importance of nonlocal electron correlation in the $\mathrm{BaNiS}_{2}$ semimetal from quantum oscillations studies, Phys. Rev. B 97, 075140 (2018).

[36] P. Giannozzi, S. Baroni, N. Bonini, M. Calandra, R. Car, C. Cavazzoni, D. Ceresoli, G. L. Chiarotti, M. Cococcioni, I. Dabo, A. D. Corso, S. de Gironcoli, S. Fabris, G. Fratesi, R. Gebauer, U. Gerstmann, C. Gougoussis, A. Kokalj, M. Lazzeri, L. Martin-Samos, N. Marzari, F. Mauri, R. Mazzarello, S. Paolini, A. Pasquarello, L. Paulatto, C. Sbraccia, S. Scandolo, G. Sclauzero, A. P. Seitsonen, A. Smogunov, P. Umari, and R. M. Wentzcovitch, QUANTUM ESPRESSO: A modular and open-source software project for quantum simulations of materials, J. Phys.: Condens. Matter 21, 395502 (2009).

[37] P. Giannozzi, O. Andreussi, T. Brumme, O. Bunau, M. B. Nardelli, M. Calandra, R. Car, C. Cavazzoni, D. Ceresoli, M. Cococcioni et al., Advanced capabilities for materials modelling with QUANTUM ESPRESSO, J. Phys: Condens. Matter 29, 465901 (2017).

[38] T. Ayral, S. Biermann, P. Werner, and L. Boehnke, Influence of Fock exchange in combined many-body perturbation and dynamical mean field theory, Phys. Rev. B 95, 245130 (2017).

[39] S. Pagliara, G. Galimberti, S. Mor, M. Montagnese, G. Ferrini, M. S. Grandi, P. Galinetto, and F. Parmigiani, Photoinduced band gap renormalization in graphite, J. Am. Chem. Soc. 133, 6318 (2011).

[40] G. Gatti, A. Crepaldi, M. Puppin, N. Tancogne-Dejean, L. Xian, U. De Giovannini, S. Roth, S. Polishchuk, P. Bugnon, A. Magrez, H. Berger, F. Frassetto, L. Poletto, L. Moreschini, S. Moser, A. Bostwick, E. Rotenberg, A. Rubio, M. Chergui, and M. Grioni, Light-Induced Renormalization of the Dirac Quasiparticles in the Nodal-Line Semimetal ZrSiSe, Phys. Rev. Lett. 125, 076401 (2020).

[41] K. W.-K. Shung, Dielectric function and plasmon structure of stage-1 intercalated graphite, Phys. Rev. B 34, 979 (1986).

[42] T. Ando, Screening effect and impurity scattering in monolayer graphene, J. Phys. Soc. Jpn. 75, 074716 (2006).

[43] E. H. Hwang and S. Das Sarma, Dielectric function, screening, and plasmons in two-dimensional graphene, Phys. Rev. B 75 , 205418 (2007).

[44] E. V. Gorbar, V. P. Gusynin, V. A. Miransky, and I. A. Shovkovy, Magnetic field driven metal-insulator phase transition in planar systems, Phys. Rev. B 66, 045108 (2002).

[45] S. V. Syzranov and B. Skinner, Electron transport in nodal-line semimetals, Phys. Rev. B 96, 161105(R) (2017).

[46] I. E. Grey and H. Steinfink, Crystal structure and properties of barium nickel sulfide, a square-pyramidal nickel(II) compound, J. Am. Chem. Soc. 92, 5093 (1970). 